\title{
Nanocellulose reinforced polymer composites: Computational analysis of structure- mechanical properties relationships
}

Mishnaevsky, Leon; Mikkelsen, Lars Pilgaard; Gaduan, Andre N.; Lee, Koon-Yang; Madsen, Bo

Published in:

Composite Structures

Link to article, DOI:

10.1016/j.compstruct.2019.111024

Publication date:

2019

Document Version

Peer reviewed version

Link back to DTU Orbit

Citation (APA):

Mishnaevsky, L., Mikkelsen, L. P., Gaduan, A. N., Lee, K-Y., \& Madsen, B. (2019). Nanocellulose reinforced polymer composites: Computational analysis of structure-mechanical properties relationships. Composite Structures, 224, [111024]. https://doi.org/10.1016/j.compstruct.2019.111024

\section{General rights}

Copyright and moral rights for the publications made accessible in the public portal are retained by the authors and/or other copyright owners and it is a condition of accessing publications that users recognise and abide by the legal requirements associated with these rights.

- Users may download and print one copy of any publication from the public portal for the purpose of private study or research.

- You may not further distribute the material or use it for any profit-making activity or commercial gain

- You may freely distribute the URL identifying the publication in the public portal 


\title{
Nanocellulose reinforced polymer composites: Computational analysis of structure-mechanical properties relationships
}

\author{
Leon Mishnaevsky Jr. ${ }^{1}$, Lars P. Mikkelsen ${ }^{1}$, Andre N. Gaduan ${ }^{2}$, Koon-Yang Lee ${ }^{2}$, Bo Madsen ${ }^{1}$ \\ ${ }^{1}$ Department of Wind Energy, Technical University of Denmark, 2000 Roskilde, Denmark \\ ${ }^{2}$ Department of Aeronautics, Imperial College London, South Kensington Campus, London, SW7 2AZ, United Kingdom
}

\begin{abstract}
:
Structure-mechanical properties relationships of nanocellulose reinforced polymer composites are studied in computational experiments. A code for the automatic generation of 3D unit cell finite element models of nanocellulose reinforced polymers with "snake"-shaped nanocellulose fibrils is developed. The code allows the generation of pre-defined nanocomposites structures, with varied angles between nanocellulose snakes segments and hydrogen bonds between nanocellulose fibrils. In a series of computational studies, it is demonstrated that the nanocellulose reinforcement leads to higher stiffness of the matrix polymer, but makes it more brittle.
\end{abstract}

Keywords: Biocomposites; Nanocomposites; Nanocellulose; Composites; Computational modelling

\section{Introduction}

Nanoengineering is an attractive and promising way to enhance the performance and properties of composites. In many works [e.g., 1-4], the drastic improvement of composite properties as a result of secondary reinforcement by nanoscale particles (additionally to the usual microscale fibers or particles) has been demonstrated. A very interesting and promising nanoscale material with the potential to enhance the polymer properties is the nanocellulose fibrils. As differed from often used carbon nanotubes or graphene, the nanocellulose fibrils are natural materials, widely available, and relatively cheap. In several works, hierarchical composites with nanocellulose nanoscale reinforcements have been developed [5-9].

For the computational analysis of nanocellulose (NC) composites, a number of computational models have been developed. Quite often, the models are based on common micromechanics of composite approaches (theory of laminates, rule of mixtures, Halpin-Tsai models [10, 11], Cox-Krenchel model

*Email: lemi@dtu.dk 
for randomly oriented short fibre composites [12]), but also on fibre network models (developed earlier in the framework of paper studies), like Poisson fibre network [13] and percolation models [14]. Several promising directions are connected by combining the network models with micromechanics of composite methods, like the version of rule of mixtures when representing the $\mathrm{NC} /$ polymer composite as polymer/"NC paper" composites [11], finite element model of fibre network [15] or the network model based on fibre-fibre bond model combined with rule of mixtures approach [10]. Josefsson et al. [16] developed a multiscale analytical model of cellulose nanofibril reinforced composites by using the laminate theory, self-consistent Mori-Tanaka model and micromechanical models.

In this paper, we seek to investigate the relationships between structures, mechanical properties and damage resistance of nanocellulose reinforced polymers using 3D computational simulations of deformation and damage behaviour, taking into account the real nanocellulose structures, and hydrogen bonding between the nanofibrils. We consider first of all the case of polymers with relatively low content of nanocellulose fibrils, studying both to the "homeopathic effect in nanocellulose reinforced polymer composites as often observed for carbon nanotube reinforced polymers" [11], and the mechanisms controlling the reinforcing effect of nanocellulose. In order to carry out such simulations, a new software for the automatic generation of 3D unit cell finite element models of nanocellulose reinforced polymers with "snake"-shaped nanocellulose fibrils was developed. Varying the structures of nanocomposites (content of nanocellulose, availability of hydrogen bonds, etc), we investigate the relationships between structures and the damage behavior.

\section{Computational modelling of nanocellulose composites}

\subsection{Nanostructural features of nanocellulose reinforced composites}

The micromechanical structural models of nanocomposites are usually based on the (older) models of common composites (see review in [17]), however, taking into account peculiarities of nanoscale structures. Among these peculiarities, one should mention high aspect ratio of nanoparticles, large surfaces, and interface effects [18]. These aspects of nanocomposite modelling have been discussed elsewhere $[18,19,20]$. With view on nanocellulose, there are a number of other peculiarities as compared with other artificial nanoparticles. In the case of carbon nanoparticles or nanoclay, the mechanical constraining of polymer chains, and surface/polymer interaction (with formation of layer of perturbed polymers around nanoparticles) are supposed to be responsible for extraordinary effect of nanoparticles on the mechanical properties. In the case of nanocellulose, different mechanisms allow the nanocellulose to influence and enhance the polymer properties, among them, formation of 
network of long nanoscale fibers and strong hydrogen bonds between nanocellulose fibrils. As different from carbon nanoparticles, nanocellulose fibrils do not form clusters but percolation networks which have positive effect on strength of composites [11]. The interaction between nanoparticles is controlled not only by Van der Waals forces but also by hydrogen bonds forming between nanocellulose fibrils [21]. The adhesion to the polymer matrix is dominated by hydrogen bonding [21]. While nanocellulose fibrils can be assumed to have snake-like shapes (see Figure 1) (similarly to carbon nanotubes, as shown in [22]), these shapes are even less regular. In [23], the zigzagged shapes of nanocellulose fibrils were observed. These nanocellulose fibrils have average length of $2000 \mathrm{~nm}$, radius of $6 \mathrm{~nm}$ and on average 6 kinks ("turns") of the snake, each at the angle of up to $60^{\circ}$ to the previous segments [24].

\subsection{Software for the automatic generation of finite element models of nanocellulose reinforced polymer composites}

For the efficient computational testing of various structures, a new computational code for the automatic generation of 3D unit cell models of nanocomposites was developed. The program, written in Fortran, generates a Python code, which is running in the finite element software Abaqus, and generates FE models with pre-defined parameters.

The simulations are performed by the commercial FE code ABAQUS implicit. The mesh is generated with tetrahedral element C3D10 and linear brick element, with reduced integration C3D8R by the free mesh control.

Main parameters. A cube shaped unit cell is created with the side size $500 \mathrm{~nm}$. The initial parameters as volume content of NC (varied), fibril length $(2000 \mathrm{~nm})$, fibril radius $(3 \mathrm{~nm})$, and the number of "kinks" per NC fibril (varied) are defined. The length of each NC fibril segment is the length of the fibril divided by the number of "kinks" (which can be varied in the model).

Generation of snake-shaped fibrils. The main challenge here was to implement realistic shape features in the computational models, instead of idealized "cylindrical" nanoparticles [11]. It was realized as follows. The coordinates of initial point of each $\mathrm{NC}$ fibril was given by three random numbers inside the cell, determined using the random number generator. Then the next point is defined, by defining (again randomly) two angles ( $\alpha$ and $\beta$, in the range from 0 to 360 degrees) as shown in Figure 2. The location of each following turning point is defined by newly generated random angles (now, in the range of $-60^{\circ}$ to $60^{\circ}$ ). After the points were generated, wires were plotted through the points, and round sections of given diameter were swept through each array of points.

Arrangement of nanoparticles. The Random Sequential Adsorption approach (RSA) for the snakeshaped inclusions was realized. This approach consists in a sequential addition of randomly oriented reinforcements to a unit cell, ensuring that the fibrils (and also segments of fibrils) do not overlap 
$[19,25]$. The distances between newly generated points and all the available points were checked, and if the points were at the same place, too close or out of box, the point was generated again (using the RSA algorithm). Furthermore, if the line segments (newly generated segment and old segments) touched, the second point of the new line segment was generated again as well. The software developed is based on the algorithm from [19]. Figure 3 shows examples of generated assemblies of nanocellulose fibrils.

Meshing of the complex unit cell. In the developed program, two options were included: geometrybased meshing (GBM), and embedding element meshing approach (EEM). The first option works well for not too complex structures ("thick" nanocellulose fibrils, with less kinks and low zigzagging angles). For the more complex structures in the present study (with large fibril aspect ratio of about 300 , and large number of kinks), this approach will lead to meshing problems, or, if a mesh is generated, to simulation problems. For this case, the developed software offers the EEM option, where the structures with very fine meshes are embedded into regular meshes of the unit cell.

Introducing the hydrogen bond linkage into the 3D FE model. In order to simulate the effect of hydrogen bonds between the nanocellulose fibrils in the 3D model, new subroutines were added to the previously developed code. After the locations of all the points of the NC "snakes" are defined, the distances between each of the segments are calculated using the algorithm by Vega and Lago [26]. Distances between the segments of the same NC fibril are not calculated. If the distance between the segments is lower than a given value, it is assumed that hydrogen bonds are formed between the points. Then, a bond (cylindrical "secondary snake") is created between the end points of these segments, with the assumed radius $0.5 \mathrm{~nm}$. The bonds between the points are slightly curved, to simplify the geometrical model generation. Several alternative approaches were tested, including placing reference points on the "snakes", creating connectors and ties between points, and so on, however, all these (constraints-based) approaches, which work pretty well in the case of "no matrix" model of materials with many interconnected fibers [27], led to the over-constraining in the model with the fibers embedded into the matrix. Thus, the hydrogen bonds were realized as solid "physical" bonds between the fibrils, instead of introducing additional constraints.

\section{3 "Window" and "periodic" unit cell design}

The material Representative Volume Element, RVE, is a cube of matrix which contains nanoreinforcements. The code developed allowed two options for unit cells: (a) "window" type and (b) "periodic" type (see Figure 2). In the first option, the "window" type unit cell, the nanocellulose fibrils are constrained inside the cube. If a segment of a nanocellulose snake extends to a point outside the cube, the program rejects this point and requires the code to generate the segment direction again (again, with the random number generator). In the second option, the "periodic" type 
unit cell, the segments of snakes are allowed to go out of the cube, but are cut at the cube faces, and are then continued (in the same direction) from the opposite face of the cube. The advantages and disadvantages of these two types of unit cells are discussed elsewhere [17]. The second option is considered as better reflecting the real structure of materials.

\subsection{Materials properties, damage and porosity}

In this work, PMMA reinforced with nanocellulose fibrils is considered. The mechanical properties are as follows: Young's modulus $[\mathrm{GPa}]=2.68$, Poisson's ratio $=0.30$. For nanocellulose fibrils, a Young's modulus of $140 \mathrm{GPa}$ and a Poisson's ratio of 0.30 were taken from [15]. As a first approximation, the nanocellulose fibrils are assumed to be isotropic.

Damage modelling: The damage analysis is carried out by a User Defined Field Subroutine (USDFLD, in ABAQUS) written in FORTRAN, in which for each load-increment, and for each element, the damage criterion is compared to its critical value. As damage criterion, the local critical strain was used. The local critical strain (in a finite element) was estimated using inverse modelling, to fit the local strain (taking into account the stress localization) to the experimentally observed global failure strain (4.6\%, for PMMA). The estimated value (local critical strain $\varepsilon=0.18$ ) corresponds to the data observed in [28]. If the damage criterion reaches the critical value, the Young's modulus of the element is reduced by 1000 times.

\section{Structures-mechanical properties relationships of nanocellulose/polymer composites: Computational studies}

In this section, relationships between parameters of nanoscale structures of nanocellulose/polymer composites and their mechanical properties and strength are analysed.

In order to analyse the effect of volume content of nanocellulose on the mechanical properties and damage behaviour of nanocomposites, a number of $3 \mathrm{D}$ unit cells with varied volume contents were generated and tested. The unit cell size was $500 \times 500 \times 500 \mathrm{~nm}^{3}$. Three versions of each unit cells (with same volume contents, but different seed numbers of random number generators, leading to different nanoparticle arrangements) were generated and tested. The amount of "turns" in the nanocellulose snakes was taken 7.

Figure 4a shows typical stress-strain curves obtained in simulations for the unit cells with varied volume content of nanocellulose fibrils. Here, the hydrogen bonding effect was suppressed. In order to explore the combined effect of volume content and hydrogen bonding, a series of simulations of the unit cells with different volume contents of nanocellulose and hydrogen bonding were carried 
out. Figure $4 \mathrm{~b}$ shows the stress-strain curves for unit cells with suppressed and non-suppressed hydrogen bonding. In these simulations, the hydrogen bonding was assumed to take place when the distance between segments is smaller than $100 \mathrm{~nm}$. The effect of the range of hydrogen bonding on the mechanical properties and damage behavior of the nanocellulose reinforced composites was also studied in the numerical experiments. The critical distances between snake-shaped nanocellulose fibres were varied (30,100 and $150 \mathrm{~nm}$ ), leading to 5,222 and 568 bonds between the fibres in the model (with $1 \%$ of nanocellulose). Figure 5 shows the peak stress of the nanocomposites plotted versus the hydrogen bonding radius

From these curves, it can be seen that the nanocellulose fibrils increases the stiffness of the polymer but this is also accompanied by a drastic decrease in the failure strain. This is due to stress concentration and damage initiation on the nanocellulose ends, which triggers the damage growth and failure of the nanocomposite. Figure 6 shows the damaged finite elements in the material with increasing loading. It can be seen that the microcracks form first at the ends of nanocellulose fibrils. Then, they grow both at the ends, and along the snakes, most intensively in the regions of "turns". Further, the cracks grow intensively between the close nanoparticles, linking the cracks on the fibrils. From Figure $4 \mathrm{~b}$, it can be seen that the availability of hydrogen bonds delays the damage initiation, slowing down the damage evolution in polymer, slightly increases the failure strain and to some degree reduces the brittleness of nanocellulose reinforced polymers. Apparently, the additional bonds make all the structure more constrained.

\section{Comparison with experimental data and literature}

\subsection{Comparison with experiments}

For the validation of the numerical results, a series of experiments has been carried out. Composite samples with $=$ poly $($ methyl methacrylate $)($ PMMA $)$ matrix and bacterial nanocellulose $(B C)$ were manufactured as follows.

Materials. Acetone (GPR RECTAPUR, purity > 99.5\%) and sodium hydroxide $(\mathrm{NaOH})$ (pellets, AnalaR NORMAPUR ${ }^{\circledR}$, purity $>98.5 \%$ ) were purchased from VWR International Ltd (Lutterworth, UK). PMMA powder (200 $\mu \mathrm{m}$, MW $\sim 75 \mathrm{kDa}$, PDI $\sim 2.8$ ) was purchased from Polysciences Europe $\mathrm{GmbH}$ (Hirschberg, DE) and used as the polymer matrix. BC was extracted from commercially available nata de coco cubes (Coconut gel in syrup, Xiangsun Ltd, Lugang Township, Changhua County, Taiwan). BC was used in this study as BC is pure celluose without impurities, such as residual lignin and hemicellulose that are often present in plant-derived nanocellulose. 
Purification of $\mathrm{BC}$ from nata de coco cubes. The purification of $\mathrm{BC}$ from nata de coco follows our previous work in batches of $150 \mathrm{~g}$ of nata de coco cubes. Firstly, the cubes were stirred in $3.5 \mathrm{~L}$ of de-ionised water and heated to $80^{\circ} \mathrm{C}$. $\mathrm{NaOH}$ pellets $(14 \mathrm{~g})$ were added and the suspension was left to stir for $2 \mathrm{~h}$. After this step, the purified cubes were recovered by pouring the content onto a metal sieve to drain away the $\mathrm{NaOH}$ solution. The cubes were then rinsed with de-ionised water, prior to blending in another $5 \mathrm{~L}$ of de-ionised water for 2 min to produce a homogeneous $\mathrm{BC}$ suspension. The $\mathrm{BC}$ suspension was then centrifuged at $7000 \mathrm{~g}$ for $6 \mathrm{~min}$ to remove the excess water. This blendingcentrifugation step was repeated until a neutral $\mathrm{pH}$ was attained. The consistency of BC-in-water was then adjusted to $1 \mathrm{~g} \mathrm{~L}^{-1}$, flash frozen in liquid nitrogen and freeze-dried prior to subsequent use.

Production of neat PMMA and model BC-reinforced PMMA composites. Model BC-reinforced PMMA composites containing 0.5 vol.-\%, 1.0 vol.-\% and 1.5 vol.-\% BC in PMMA was prepared in this work. Briefly, freeze-dried BC was first dispersed in $100 \mathrm{~mL}$ of acetone using a hand blender. 20 $\mathrm{g}$ of PMMA powder was then added into the BC-in-acetone suspension and magnetically stirred overnight to dissolve the PMMA. The BC-polymer solution was then casted onto a glass plate using an automatic film applicator (Model 3430, Elcometer Ltd., Manchester, UK) and the solvent was left to evaporate at room temperature for 2 days. As a control, $20 \mathrm{~g}$ of PMMA was dissolved in $50 \mathrm{~mL}$ of acetone under magnetic stirring prior to solution casting. The solution casted PMMA and BC-PMMA films were then placed in a vacuum oven at $50^{\circ} \mathrm{C}$ to further evaporate the solvent, prior to compression moulding using a heated hydraulic press operating at a compaction force and temperature of 2 tonnes and $155^{\circ} \mathrm{C}$, respectively. The thickness of the resulting neat PMMA and model BC-reinforced PMMA composites were measured to be $\sim 250 \mu \mathrm{m}$.

Porosity of neat PMMA and model BC-reinforced PMMA composites. The density $\rho$ of the neat PMMA and model BC-reinforced PMMA composites was measured using He pycnometry (Accupyc II 1340, Micromeritics Ltd. Hexton, UK). The apparent density $\rho_{\mathrm{e}}$ of the samples was calculated from the mass and apparent volume of the samples. The porosity $P$ was then calculated using: $\mathrm{P}$ [\%] $=\left(1-\rho / \rho_{\mathrm{e}}\right) \times 100 \%$.

Tensile properties of neat PMMA and model BC-reinforced PMMA composites. Prior to the test, dog bone shape test specimens, with an overall length of $35 \mathrm{~mm}$, a gauge length of $10 \mathrm{~mm}$ and a width of $2 \mathrm{~mm}$, were cut using a manual punch die (ZCP020, Zwick Testing Machines Ltd., Leominster, UK). Tensile test was then carried out on a micro-tensile tester (Model MT-200, Deben UK Ltd, Woolpit, UK) equipped with a $200 \mathrm{~N}$ load cell. The strain of the test specimens was evaluated using a non-contacting video extensometer (iMetrum Ltd, Bristol, UK). The tensile tests 
were conducted at a crosshead displacement speed of $1 \mathrm{~mm} \mathrm{~min}^{-1}$. Average results of 5 test specimens were reported for each sample.

\subsection{Comparison with experiments and correction on porosity. Literature data}

The tensile properties of neat PMMA and model BC-reinforced PMMA composites are summarised in Table 1 and their respective stress-strain curves are shown in Figure Error! Reference source not found.. When $\mathrm{BC}$ was used as reinforcement for PMMA, the tensile modulus increased from 3.0 GPa for neat PMMA to $3.2 \mathrm{GPa}$ for 0.5 vol-\% and 1.0 vol.-\% BC-reinforced PMMA composites. Increasing the $\mathrm{BC}$ loading to 1.5 vol-\% further increased the tensile modulus of the resulting $\mathrm{BC}$ reinforced PMMA composites to 3.6 GPa. These results showed that $\mathrm{BC}$ acts as excellent stiffening agent for polymers. However, BC does not seem to improve the tensile strength of the resulting composites; no significant difference in the tensile strength could be observed. All BC-reinforced PMMA composites possessed similar tensile strength of $\sim 47 \mathrm{MPa}$, independent of the loading fraction of BC, similar to that of neat PMMA of $\sim 44 \mathrm{MPa}$. Nevertheless, the introduction of BC into PMMA led did increase the strain-at-break of the resulting composites. Neat PMMA was found to possess a strain-at-break of $2.3 \%$. Increasing the loading fraction of $\mathrm{BC}$ leads to a progressive decrease in the strain-at-break of BC-reinforced PMMA composites to only $1.7 \%$ (for 1.5 vol.- $\%$ BC-reinforced PMMA composites).

Comparing the experimentally and numerically obtained values, one can see that the experiments qualitatively confirm the numerical results. With increasing the nanocellulose content from $0.5 \%$ to $1 \%$ and then to $1.5 \%$, the strain at failure decreases by $8 \%$ in both the model and experiment, and then by $16 \%$ and $11 \%$ for model and experiments.

However, for both Young modulus and strength, the computational model clearly overestimate the effect of nanocellulose: while the experimental data show the increase of Young modulus by 5, 7 and $18 \%$ at the volume content of nanocellulose $0.5,1$ and 1.5\%, the computational model estimations are 10,35 and $71 \%$. The model above does not include the growing porosity of the material, with increasing the nanocellulose particles content. In the table 1, one can see that the porosity of the material grows drastically, from $2.7 \%$ at $0.5 \%$ of nanocellulose, to $5.2 \%$ at $1.5 \%$ of nanocellulose.

The increase of the nanocomposite porosity with increasing the density of nanofibrils is related to the limited fibre spacing for matrix impregnation, leading to the development of porosity in the composites, beyond a certain transition fibre volume fraction [29]. As noted in [29], such impregnation porosity, which in fact includes matrix and interface porosity has a strong effect on the 
mechanical properties of the material. Further, a linear relationship can be established between porosity content and nanofibre content [31].

In order to estimate the effect of porosity on the elastic properties of nanofibril reinforced polymer, a simple 3D unit cell models have been developed. A model included a single segment of the nanofibril, and a given amount of spherical pores. Following [30], we assume the pore size in PMMA/nanofibril composites as $10 \mathrm{~nm}$. Figure 7 shows the unit cell model design (a) and von Mises stress distribution in the unit cell without pores (b) and with several pores (c). As expected, the stress concentration is much higher, due to the pores. Assuming that the pores don't overlap, the stiffness of the porous materials is reduced by approximately $2.34 \%$ with each additional $1 \%$ of pores. Local stresses increase drastically, when the material is porous.

Introducing the correction on the porosity in the model, and linear porosity - nanocellulose content relationship [31], one can see that the model (with porosity correction) predicts the Young modulus increased by 3,18 and $39 \%$ at $0.5,1$ and $1.5 \%$ of nanocellulose (still, larger than the experimental data).

In [35], it was observed that the addition of nanocellulose increased the elastic modulus of epoxy by $50 \%$ ", but decreased the ultimate failure stress and strain by a substantial amount. The cellulose aerogel network created stress concentrations in the epoxy system at the weak bonding sites at cellulose agglomerations. The van der Waals interaction at the cellulose associations are not strong enough to endure the stress and strain the cured epoxy can alone." The drastic, higher than expected on the basis of the rule-of-mixture increase of tensile modulus of polymers due to additions of nanocellulose was also reported in [35-37].

Sabo et al. and Ruiz et al. $[33,34]$ noticed that the reinforcement benefits of nanocellulose are linked to the interactions between the CNCs and epoxy network and the percolating network linked by $\mathrm{H}$ bonds between the NFC.

\section{Conclusions}

In this work, the structure- mechanical properties relationships of nanocellulose reinforced polymers are studied using the computational micromechanical modelling and numerical experiments. Several new computational tools and models were developed for the analysis of structure-mechanical properties relationships of the $\mathrm{NC} /$ polymer composites. A code for the automatic generation of $3 \mathrm{D}$ unit cell models with many snake-shaped nanoparticles of varied size, structure, length and number of NC fibrils is developed. 
A series of computational investigations of the deformation behaviour of nanocellulose reinforced composites is carried out. The nanocellulose fibrils (at the considered, very low volume contents) ensure some increase in the material stiffness $[19,20]$. The availability of hydrogen bonds delays the damage initiation, slows down the damage evolution in polymer, slightly increases the failure strain and to some degree reduces the brittleness of nanocellulose reinforced polymers.

Thus, the developed model reflects qualitatively the tendencies of the influence of nanocellulose on the strength and stiffness of polymers. The quantitative results vary between our experiments and results observed in literature.

Acknowledgement: The authors gratefully acknowledge the financial support of the European Commission in the framework of the EU FP7 project InCom, Grant agreement n ${ }^{\circ}$ FoF NMP2013-10608746. L.M. gratefully acknowledges also the financial support of the Innovation Foundation of Denmark in the framework of the Grand Solutions project DURALEDGE, Durable leading edges for high tip speed wind turbine blades, File nr.: 8055-00012A, as well as the support from the EU H2020 programme via the Marie Skłodowska-Curie project Nano2Day, "Multifunctional polymer composites doped with novel $2 \mathrm{~d}$ nanoparticles for advanced applications", grant agreement No 777810 .

The raw/processed data required to reproduce these findings cannot be shared at this time as the data also forms part of an ongoing study.

\section{References:}

1. Rafiee MA, Rafiee J, Srivastava I, Wang Z, Song H, Yu Z-Z, et al. Fracture and Fatigue in Graphene Nanocomposites. Small 2010;6:179-83. doi:10.1002/smll.200901480.

2. Wetzel B, Rosso P, Haupert F, Friedrich K. Epoxy nanocomposites - fracture and toughening mechanisms. Engineering Fracture Mechanics 2006;73:2375-98

3. Sidorenko D, et al. Carbon nanotube reinforced metal binder for diamond cutting tools. Materials \& Design 2015;83:536-44.

4. H.W. Zhou, et al., Carbon fiber/carbon nanotube based hierarchical composites: Effect of CNT distribution on shearing strength, Composites B, 2016, 88, 201-211.

5. J. Blaker, K.Y. Lee, A. Bismarck, Hierarchical composites made entirely from renewable resources, J Biobased Materials and Bioenergy, Vol. 5, 1-16, 2011

6. I. Siró and D. Plackett, Microfibrillated cellulose and new nanocomposite materials: a review, Cellulose, 17, Issue 3, pp 459-494

7. L.A.Berglund and T. Pejs, MRS Bulletin, Vol.35, March 2010, p.201 
8. K. Okubo, T. Fujii, N. Yamashita, Improvement of interfacial adhesion in bamboo polymer composite enhanced with micro-fibrillated cellulose. JSME Int J, Ser A 2005; 48:, 4, pp. 199-204

9. M. Pommet, J. Juntaro, J.Y.Y. Heng, A. Mantalaris, A.F. Lee, K. Wilson, et al. Surface modification of natural fibers using bacteria: depositing bacterial cellulose onto natural fibers to create hierarchical fiber reinforced nanocomposites, Biomacromolecules 9, 1643 (2008), J. Juntaro et al, Nanocellulose enhanced interfaces in truly green unidirectional fibre reinforced composites, Compos. Interfaces 14, 753 (2007).

10. Y. Aitomaki, K. Oksman, Reinforcing efficiency of nanocellulose in polymers, Reactive \& Functional Polymers : 85/ SI, pp. 151-156 2014

11. Lee, K-Y., Aitomäki, Y., Berglund, L. A., Oksman, K., \& Bismarck, A. (2014). On the use of nanocellulose as reinforcement in polymer matrix composites. Composites Science and Technology, 105, 15-27

12. Bhagwan F. Jogi et al, Dispersion and Performance Properties of Carbon Nanotubes (CNTs) Based Polymer Composites: A Review, Journal of Encapsulation and Adsorption Sciences, 2012, 2, 69-78

13. Aström. Elasticity of Poissonian fiber networks. The American physical society, 2000,61/5

14. Favier V, Canova GR, Shrivastava SC, Cavaille JY. Mechanical percolation in cellulose whisker nanocomposites. Polym Eng Sci. 1997;37(10):1732-1739

15. Th. Denoyelle, Mechanical properties of materials made of nano-cellulose, KTH Degree, 2011

16. G. Josefsson et al., Stiffness contribution of cellulose nanofibrils to composite materialsInt J Solids and Structures, 51/2014, 945

17. L. Mishnaevsky Jr, Computational Mesomechanics of Composites, Wiley, 2007, 280 pp.

18. H.W. Wang, H.W. Zhou, R.D. Peng, L.Mishnaevsky Jr., Nanoreinforced polymer composites: 3D FEM modeling with effective interface concept, Composites Scie \& Technol, Vol. 71, No. 7, 2011, pp- 980-988

19. A.Pontenfisso, L. Mishnaevsky Jr., Nanomorphology of graphene and CNT reinforced polymer and its effect on damage: Micromechanical numerical study, Composite B (2016), pp. 338-349

20. G.M. Dai, L. Mishnaevsky Jr , Carbone nanotube reinforced hybrid composites: computational modelling of environmental fatigue and usability for wind blades, Composites Part B (2015), pp. 349-360

21. D. Gardner et al, Adhesion and Surface Issues in Cellulose and Nanocellulose Journal of Adhesion Science and Technology 22 (2008) 545-567

22. D. Sidorenko, L. Mishnaevsky Jr., E. Levashov et al, Carbon nanotube reinforced metal binders for diamond cutting tools, Materials and Design, 83, 2015, pp. 536-544

23. I.Usov et al, Understanding nanocellulose chirality and structure-properties relationship at the single fibril level, Nature Communications volume 6, Article number: 7564 (2015) 
24. EU FoF.NMP.2013-10 project INCOM. Report Deliverable D6.5 Computational simulations of nanocellulose reinforced polymers, 2016, $19 \mathrm{pp}$.

25. G.M. Dai, L. Mishnaevsky Jr., Damage evolution in nanoclay-reinforced polymers: a threedimensional computational study, Composites Science \& Technology, 74 (2013) 67-77

26. C. Vega and S. Lago A fast algorithm to evaluate the shortest distance between rods", Computers \& Chemistry 18 pp. 55-59 (1994)

27. L. Chapelle, Characterization and modelling of the mechanical properties of mineral wool, $\mathrm{PhD}$ thesis, May 2016, DTU

28. S. Zike, et al, Experimental determination of the micro-scale strength and stress-strain relation of an epoxy resin, Materials \& Design, vol: 98, pages: 47-60, 2016

29. B. Madsen et al, Plant fibre composites - porosity and volumetric interaction, Composites Science and Technology 67 (2007) 1584-1600

30. A. Svensson, Nanocomposites made from nanoporous cellulose fibres, Licentiate thesis School of Chemical Science and Engineering, KTH Stockholm, 2012

31. Madsen, B., Lilholt, H., Mannila, J., De Silva, R. T., \& Pasbakhsh, P. (2015). Volumetric composition of nanocomposites. In Proceedings of the 20th International Conference on Composite Materials ICCM20 Secretariat

32. Mohamed H. Gabr, K. Okubo, T. Fujii, Mechanical and Morphology Properties of Cellulose Nanocomposites, Handbook of Polymer Nanocomposites. Processing, Performance and Application, Springer, 2015

33. R C. Sabo et al, Characterization and Processing of Nanocellulose Thermosetting Composites Handbook of Polymer Nanocomposites. Processing, Performance and Application pp 265-295 | Cite as

34. Ruiz MM, Cavaille JY, Dufresne A, Gerard JF, Graillat C (2000) Processing and characterization of new thermoset nanocomposites based on cellulose whiskers. Compos Interfaces 7(2):117-131

35. J.Steele et al, Nanocellulose reinforcement of transparent composites Proceedings of SAMPE. Baltimore, MD. 21-24 May 2012.

36. A. Boldizar, C. Klason, J. Kubat, P. Naslund, P. Saha, Prehydrolyzed cellulose as reinforcing filler for thermoplastics, Int. J. Polym. Mater., 11 (4) (1987), pp. 229-262

37. H. Yano, J. Sugiyama, A.N. Nakagaito, M. Nogi, T. Matsuura, M. Hikita, et al. Optically transparent composites reinforced with networks of bacterial nanofibers, Adv. Mater., 17 (2) (2005), p. 153 


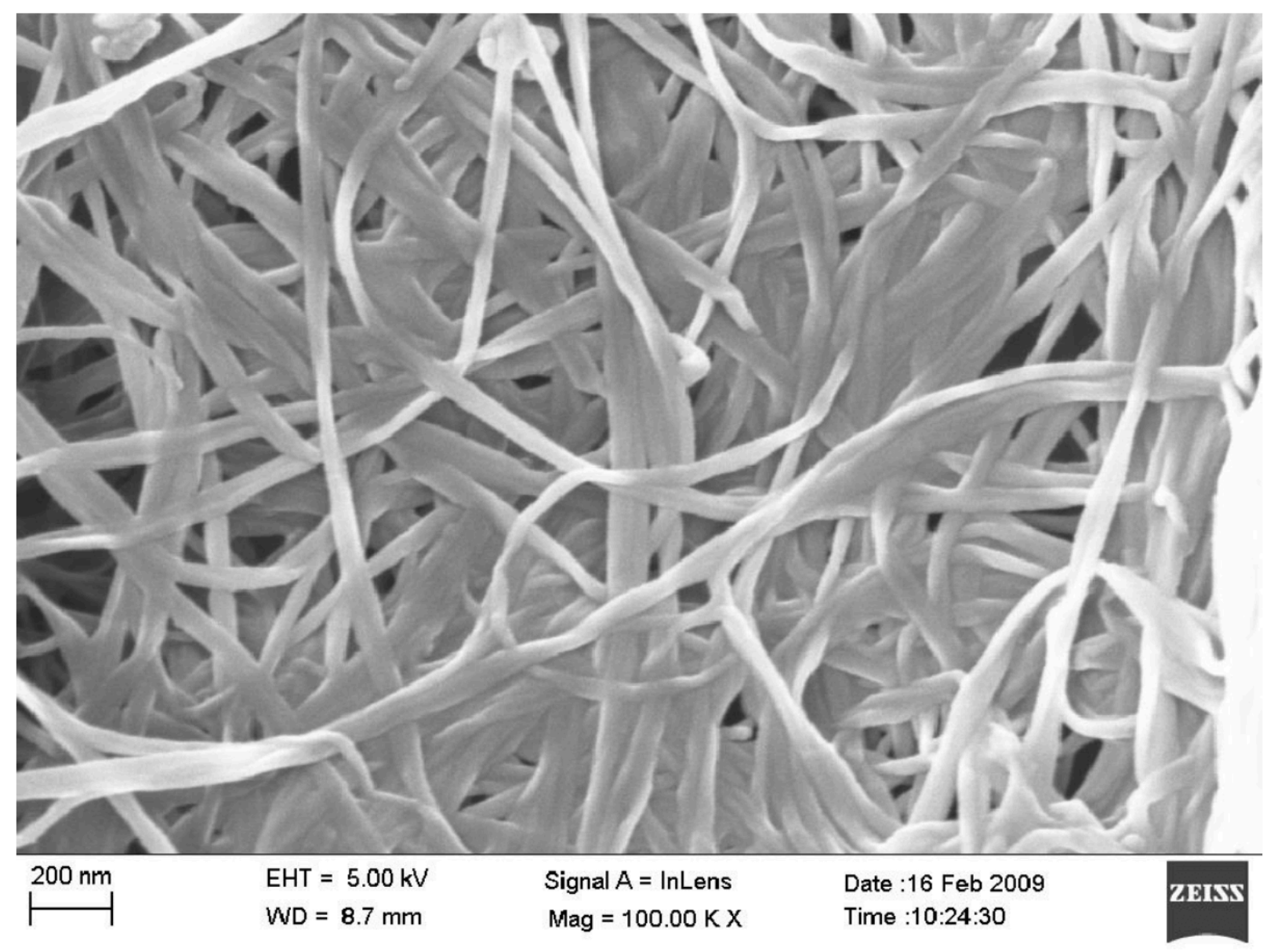

Figure $\quad$ 1. SEM Micrograph of nanocellulose fibrils 


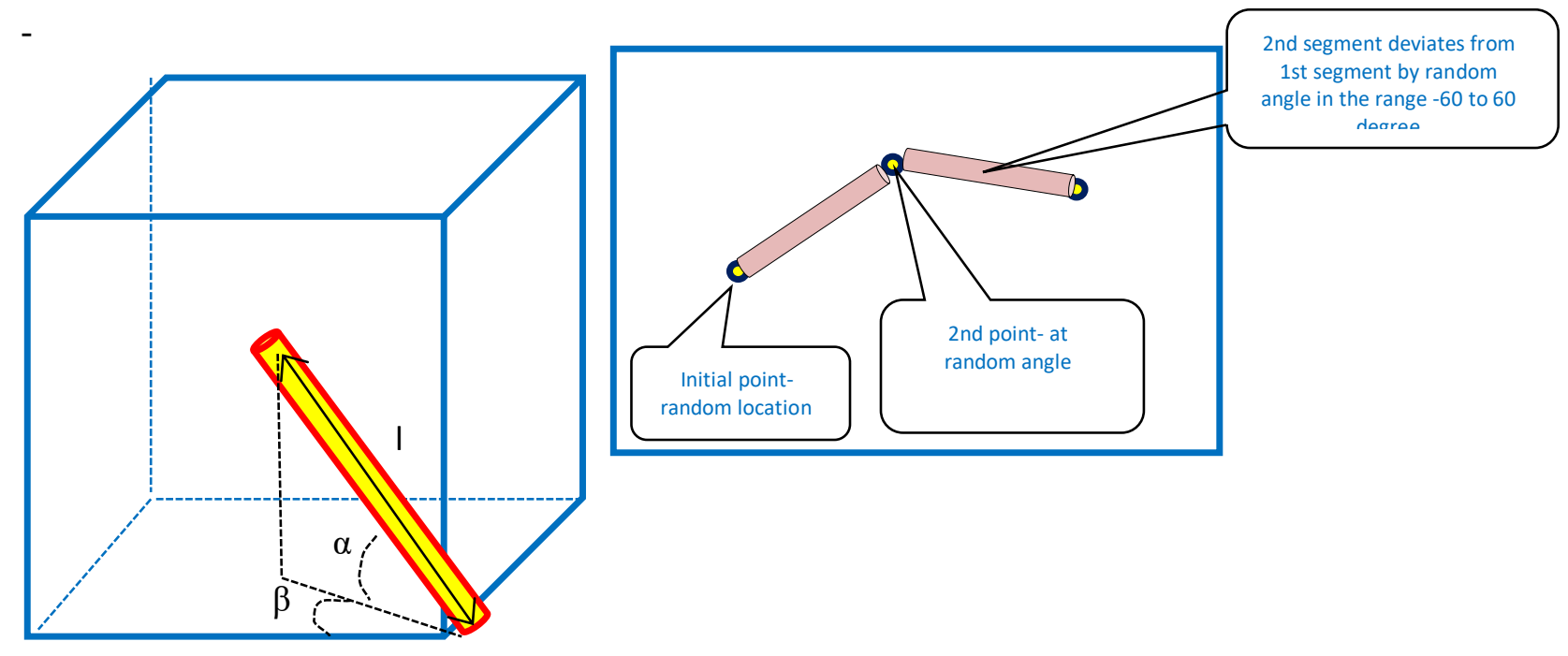

Figure

2. Schema of generation of points of the nanocellulose model

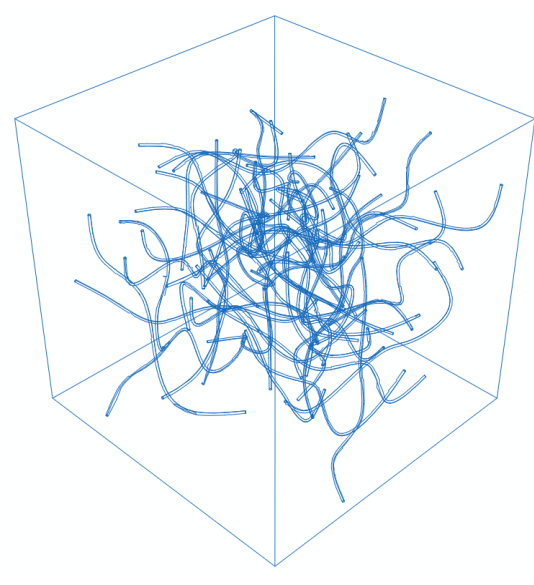

a

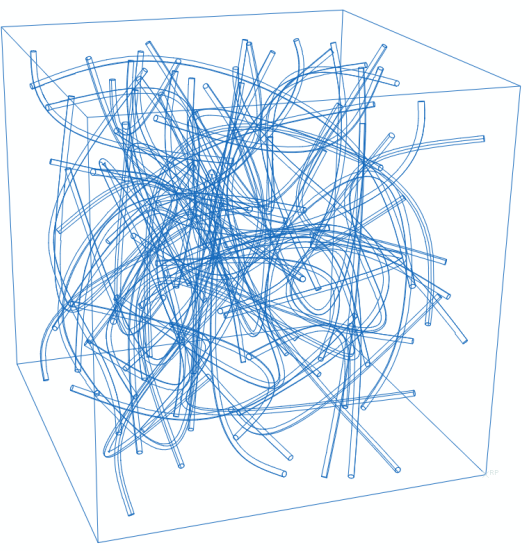

b

Figure 3. Examples of unit cells, with $30 \mathrm{NC}$ fibrils, each containing 6 kinks: (a) Windows type, (b) Periodic type. 


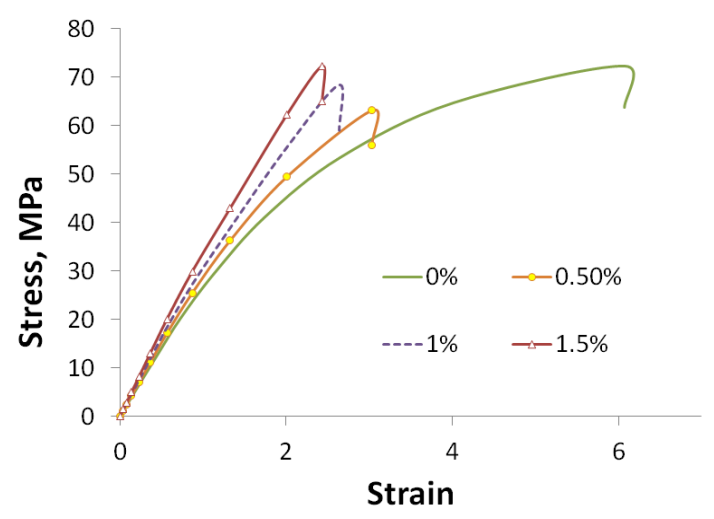

A

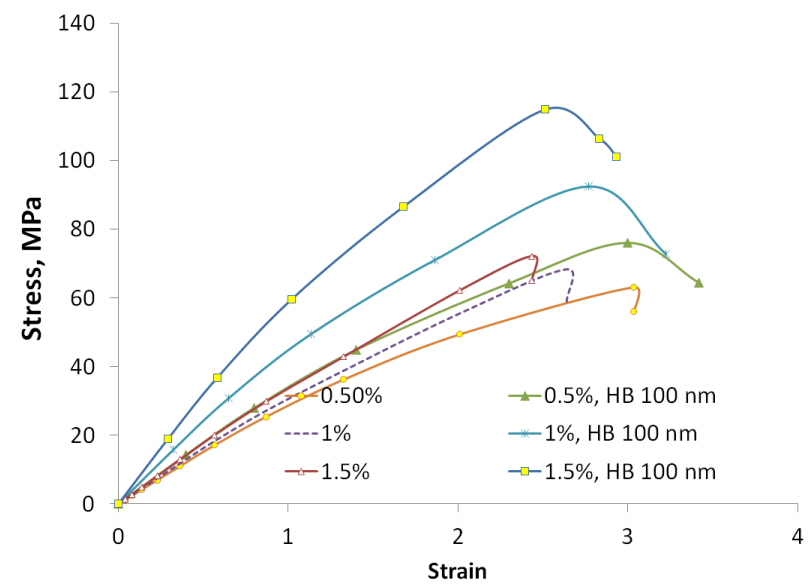

$\mathrm{b}$

Figure 4. Typical stress strain curves for unit cells with suppressed hydrogen bonding and varied NC content (a) and calculated failure strain (for the models with and without hydrogen bonding (b) (volume content varied $0 \ldots 1.5 \%$, nanofibrils 7 kicks)

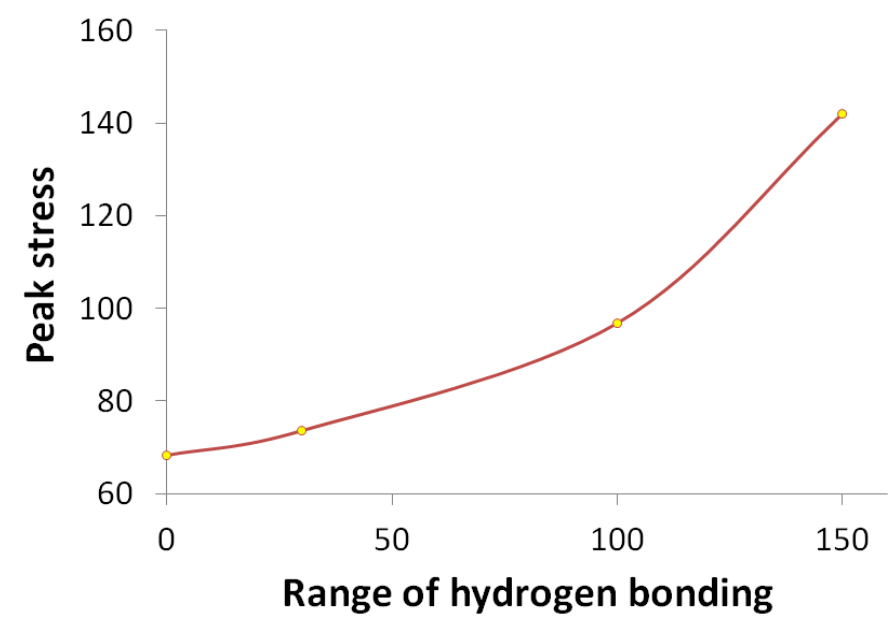

Figure 5. Peak stress (1\% content, 7 turns) plotted versus the hydrogen bonding radius 


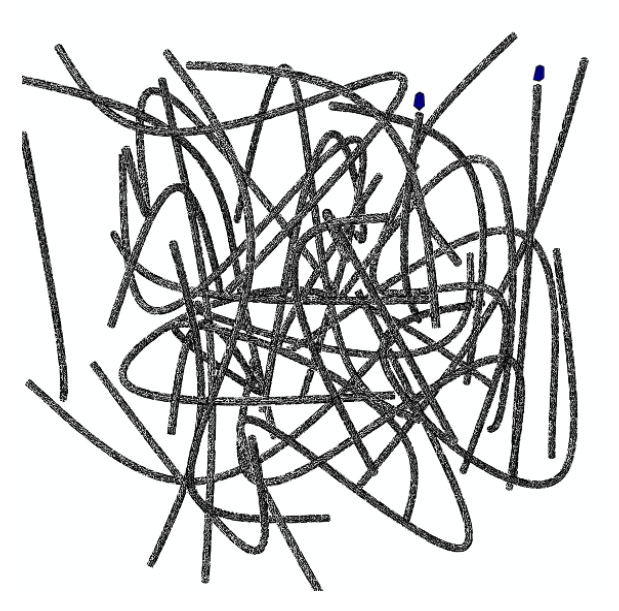

a

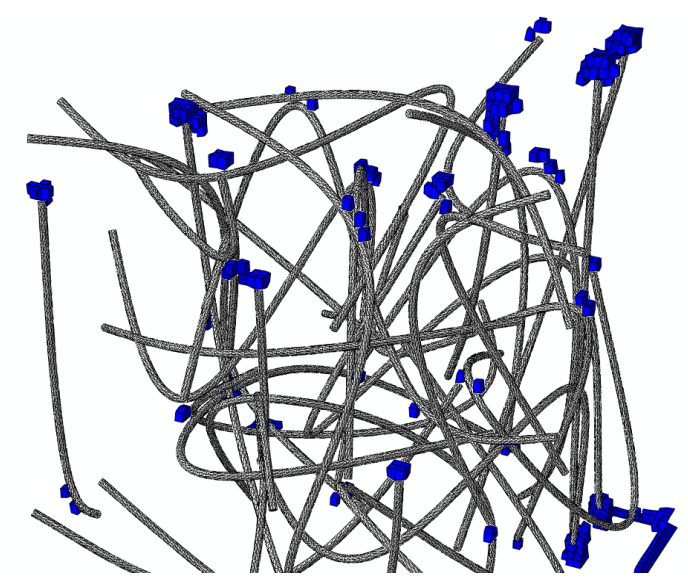

C

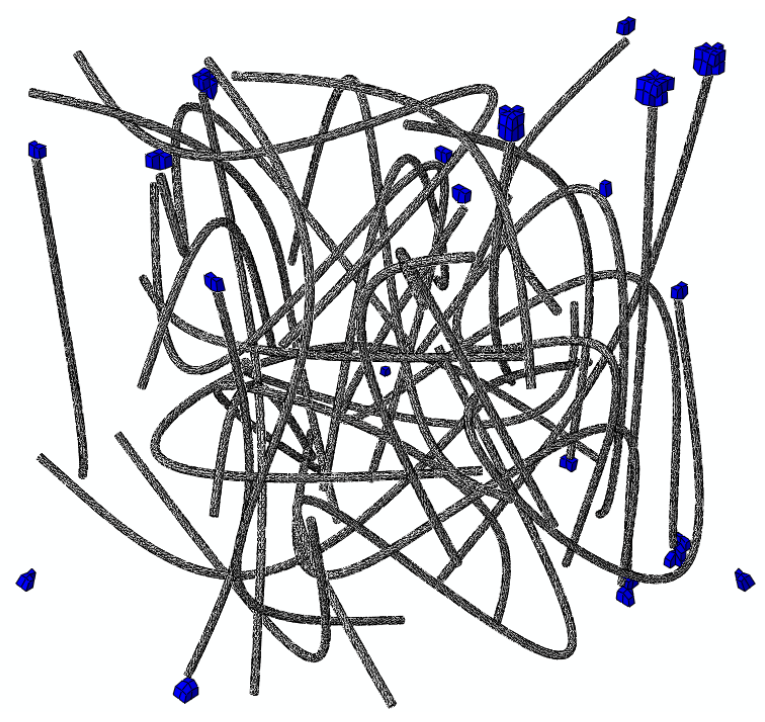

b

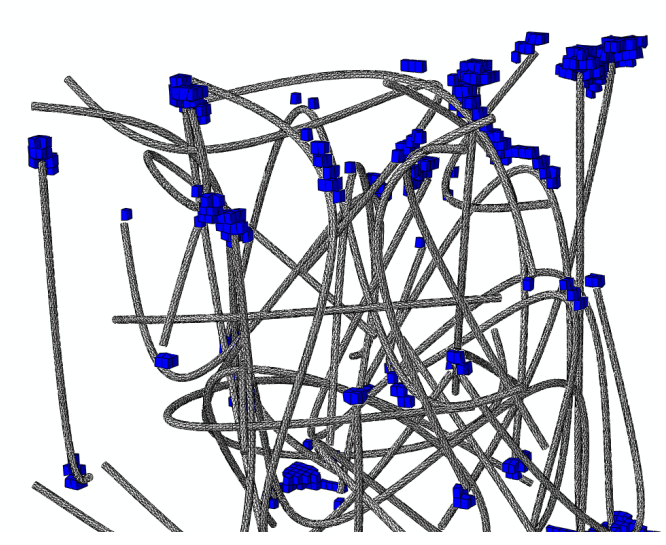

d

Figure 6. Damage development: results of simulations. $1.5 \%$ NC. 7 turns. Strain:

$0.5 \%, 0.9 \%, 1 \%, 1.1 \%$ (a-d) 


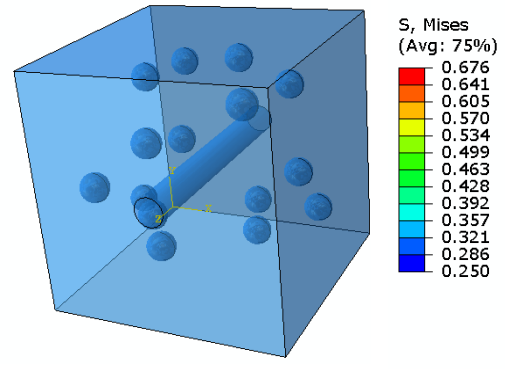

a

b
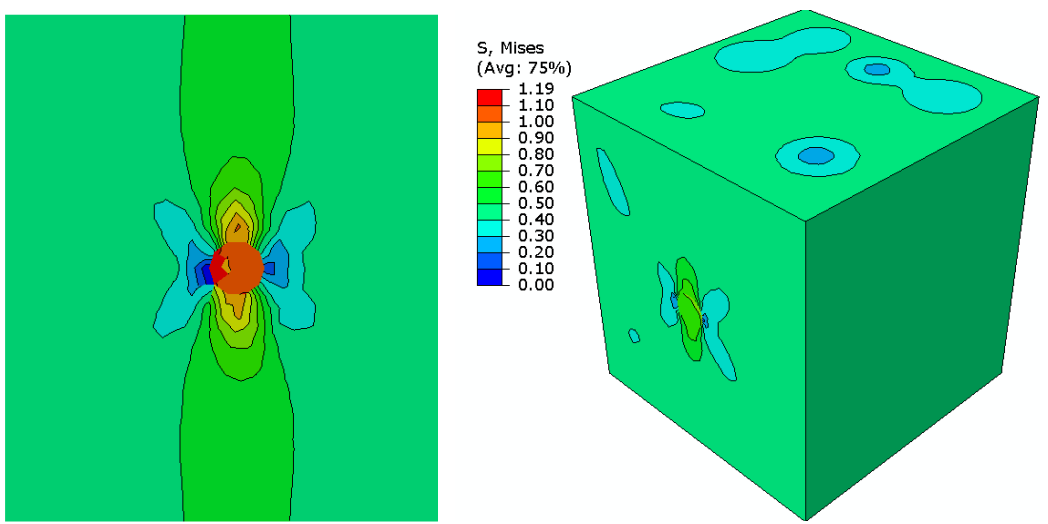

$\mathrm{c}$

Figure 7. Unit cell model of porosity around a nanofibril segment. (a) Mises stress distribution in unit cell without pores (section, $b$ ) and with several pores (c)

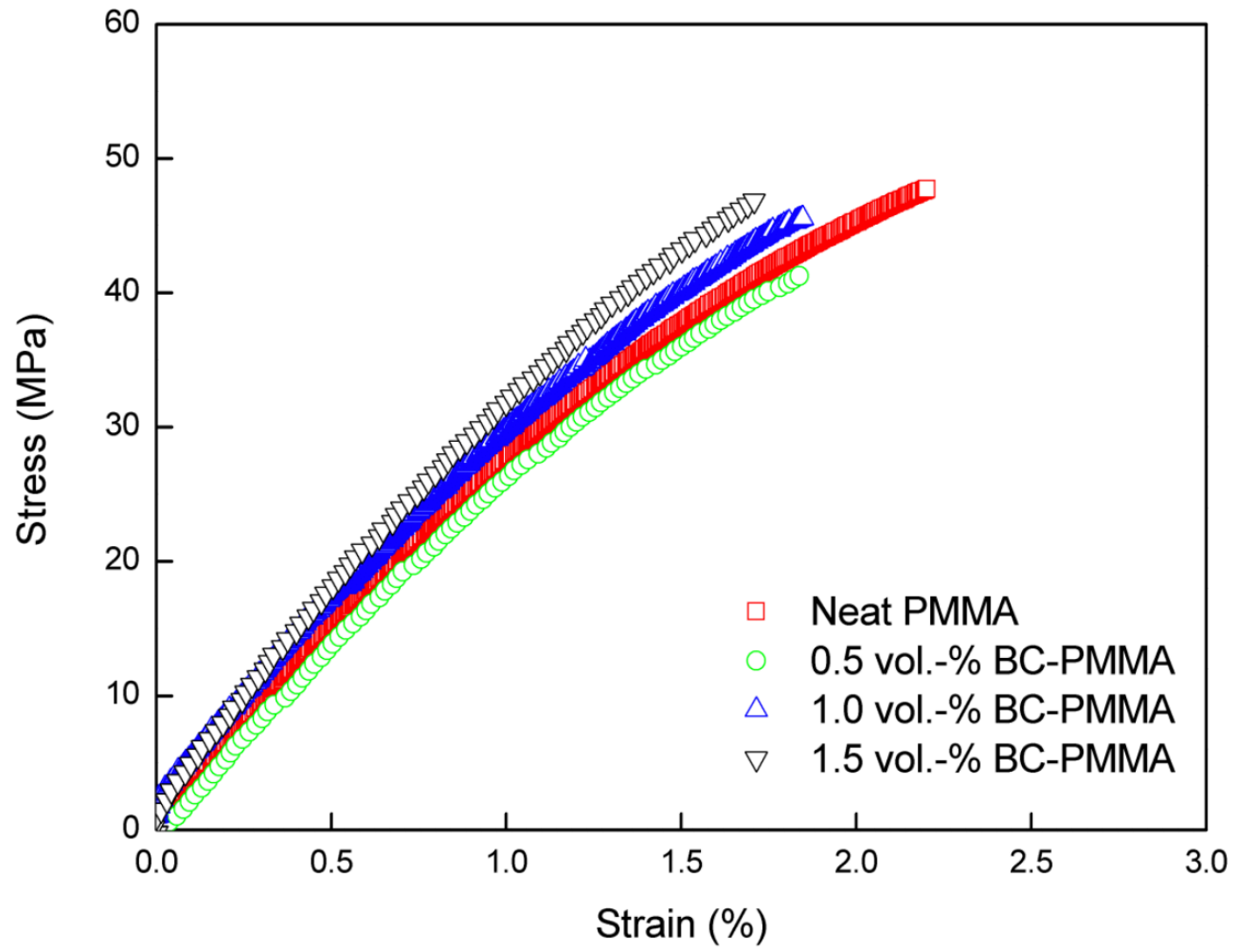

Figure $\quad 8$. Typical stress-strain curves for pure PMMA, and PMMA with bacterial nanocellulose 
Table 1. Tensile properties of neat PMMA and model BC-reinforced PMMA composites produced in this work. $\rho_{\mathrm{e}}, \rho, \mathrm{P}, \mathrm{E}, \sigma, \varepsilon$ denote the apparent density, He pynometric density, porosity, tensile modulus, tensile strength and strain-at-failure, respectively. The errors tabulated are standard errors.

\begin{tabular}{lllllll}
\hline Samples & $\rho_{\mathrm{e}}\left(\mathrm{g} \mathrm{cm}^{-3}\right)$ & $\rho\left(\mathrm{g} \mathrm{cm}^{-3}\right)$ & $\mathrm{P}(\%)$ & $\mathrm{E}(\mathrm{GPa})$ & $\sigma(\mathrm{MPa})$ & $\varepsilon(\%)$ \\
\hline Neat PMMA & $1.26 \pm 0.01$ & $1.26 \pm 0.01$ & 0 & $3.0 \pm 0.1$ & $44.4 \pm 3.1$ & $2.3 \pm 0.4$ \\
0.5 vol.-\% BC-PMMA & $1.25 \pm 0.01$ & $1.22 \pm 0.05$ & $2.7 \pm 0.1$ & $3.2 \pm 0.2$ & $47.6 \pm 4.4$ & $2.2 \pm 0.3$ \\
1.0 vol.-\% BC-PMMA & $1.28 \pm 0.01$ & $1.22 \pm 0.02$ & $4.3 \pm 0.1$ & $3.2 \pm 0.2$ & $46.1 \pm 0.9$ & $2.0 \pm 0.3$ \\
1.5 vol.-\% BC-PMMA & $1.29 \pm 0.01$ & $1.22 \pm 0.01$ & $5.2 \pm 0.1$ & $3.6 \pm 0.3$ & $47.9 \pm 3.5$ & $1.7 \pm 0.1$ \\
\hline
\end{tabular}

